\title{
Occurrence of Leaf Blight on Cosmos Caused by Alternaria cosmosa in Korea
}

\author{
Jian Xin Deng ${ }^{1}$, Ji Hye Lee ${ }^{2}$, Narayan Chandra Paul ${ }^{3}$, Hye Sun $\mathrm{Cho}^{4}$, Hyang Burm Lee ${ }^{3 *}$ and Seung Hun Yü \\ ${ }^{\prime}$ Department of Plant Protection, College of Agriculture, Yangtze University, Jingzhou 434025, China \\ ${ }^{2}$ Department of Applied Biology, College of Agriculture and Life Sciences, Chungnam National University, Daejeon 305- \\ 764, Korea \\ ${ }^{3}$ Division of Applied Bioscience \& Biotechnology, College of Agriculture \& Life Sciences, Chonnam National University, \\ Gwangju 500-757, Korea \\ ${ }^{4}$ Ginseng Resources Institute, Korea Ginseng Corp., Daejeon 305-805, Korea
}

(Received on September 24, 2014; Revised on November 24, 2014; Accepted on November 29, 2014)

In 2011, a leaf blight disease was observed on cosmos (Cosmos bipinnatus) leaves in Nonsan, Korea. The causal pathogen was isolated and identified based on morphological and molecular approaches. Morphological characteristics of the pathogen matched well with the Alternaria cosmosa and also easily distinguishable from Alternaria zinniae reported from cosmos seeds by producing branched beak. Phylogenetically, the pathogen could not be distinguished from $A$. passiflorae based on the sequence analysis of a combined data set of Alt a1 and gpd genes. However, A. passiflorae was distinguished from the present species by having conidiophores with 4 to 5 conidiogenous loci. The results indicate that the present Alternaria species is $A$. cosmosa. Pathogenicity tests revealed that the isolate was pathogenic to the leaves of Cosmos bipinnatus. This is the first report of Alternaria blight disease caused by A. cosmosa on cosmos in Korea.

Keywords : asteraceae, morphology, pathogenicity, phylogenetic analysis

Cosmos bipinnatus, known as the garden cosmos or Mexican aster, is a half-hardy annual herbaceous plant native to Mexico. The species belonging to the family Asteraceae offers flowers of solitary red, white, pink or purple color blooms (Dubey and Singh, 2011). The cosmos has been widely cultivated as a valuable ornamental plant since its first introduction in Korea in 1938 (Park, 2009). Now, it is commonly to be found in plant gardens and

*Corresponding author.

Phone) +82-42-821-5762, FAX) +82-42-823-8679

E-mail)shunyu@cnu.ac.kr, hblee@jnu.ac.kr along the sides of road or river in Korea. Fruits and aerial parts of the plant are useful as traditional herbal medicine to reduce various diseases such as jaundice, intermittent fever, and splenomegaly (Jang et al., 2008). Jang et al. (2008) reported that the cosmos flowers showed a significant antioxidant activity and protective effect against oxidative DNA damage

Anthracnose, powdery mildew and white smut diseases of Cosmos bipinnatus have been reported in Korea caused by Colletotrichum acutatum, Podosphaer fusca and Entyloma cosmic, respectively (Park et al., 2012). Cho (2004) isolated a large-spored Alternaria containing narrow-taper long beak from C. bipinnatus in Gongju and Naju, Korea, in 2000. The Alternaria species was considered as a new species based on the morphological and molecular characteristics. However, the author did not assign scientific name of the pathogen. Soon afterwards, a new species of Alternaria, A. cosmosa Y.L. Li \& W.S. $\mathrm{Wu}$ was reported from cosmos seeds in Taiwan on the basis of conidial morphology, which showed a specific pathogenicity on Cosmos bipinnatus and C. sulphureus (yellow cosmos) (Wu and Li, 2005). Simmons (2007) summarized his results of a lifetime study on Alternaria taxonomy based on morphology and described 275 species totally. But he did not include the species in his publication because he unsuccessfully obtained the extype material of $A$. cosmosa for the comparison with the other morphologically similar taxa. Meanwhile, Alternaria danida on Ageratum houstonianum is suggested as a similar taxon for $A$. cosmosa (Simmons, 2007).

In 2011, a large-spored Alternaria was isolated again from blight leaves of Cosmos bipinnatus in Nonsan, Korea. The morphological characteristics were identical to previously reported species of Alternaria (Cho, 2004). The objective of the present study was to report a new 


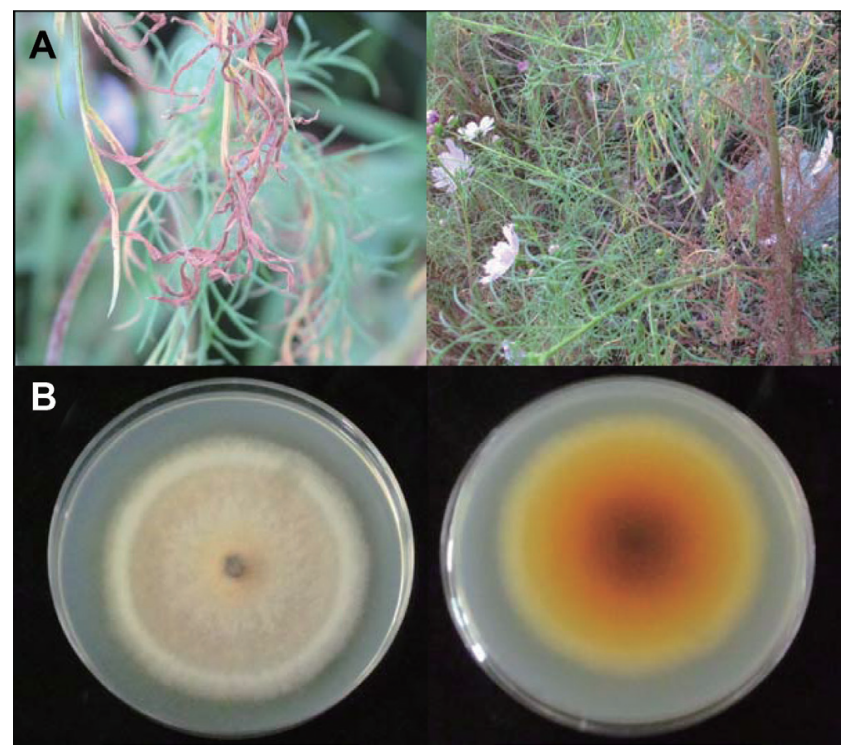

Fig. 1. Alternaria leaf blight on Cosmos bipinnatus and colony of the causal fungus. (A) discolored and necrotic symptoms on leaves of C. bipinnatus. (B) obverse (left) and reverse (right) of colony grown on potato dextrose agar at $25^{\circ} \mathrm{C}$ for 7 days.

Alternaria disease of Cosmos bipinnatus in Korea and to identify the causal pathogen based on morphological and molecular approaches.
Symptoms and isolation of the fungus. Blight symptoms on lower leaves of Cosmos bipinnatus were observed during the period of August-September 2011 in Nonsan, Chungnam, Korea (Fig. 1A). Infected leaves showed small and brown necrotic spots along with leaf discoloring. The necrotic lesions were enlarged gradually, finally resulting in the leaf blight and defoliation. The diseased leaves were cut and placed in a moist Petri dish for conidial sporulation. Pure cultures (including CNU 111459) of the Alternaria species were obtained by single spore isolation method. Another isolate (CNU 3437) of Alternaria sp. collected in 2000 (Cho, 2004) and preserved in the Culture Collection of Chungnam National University (CNU) in Korea was also used in this study. The new isolates were deposited in the CNU Culture Collection, and the Korean Agricultural Culture Collection (KACC) in Suwon, Korea.

Morphological characteristics. The isolate CNU 111459 was grown on potato dextrose agar (PDA; Difco, Montreal, Canada) incubated at $25^{\circ} \mathrm{C}$ in the dark. Colony diameters were measured after 7 days of incubation and other characteristics such as texture, color and pigmentation were also recorded. The isolate was also cultured on potato carrot agar (PCA: $20 \mathrm{~g}$ white potato, $20 \mathrm{~g}$ carrot, and 20 g agar in 1 1) under fluorescent lights (8/16 light/dark) at $22^{\circ} \mathrm{C}$ to induce sporulation (Simmons, 2007). Randomly

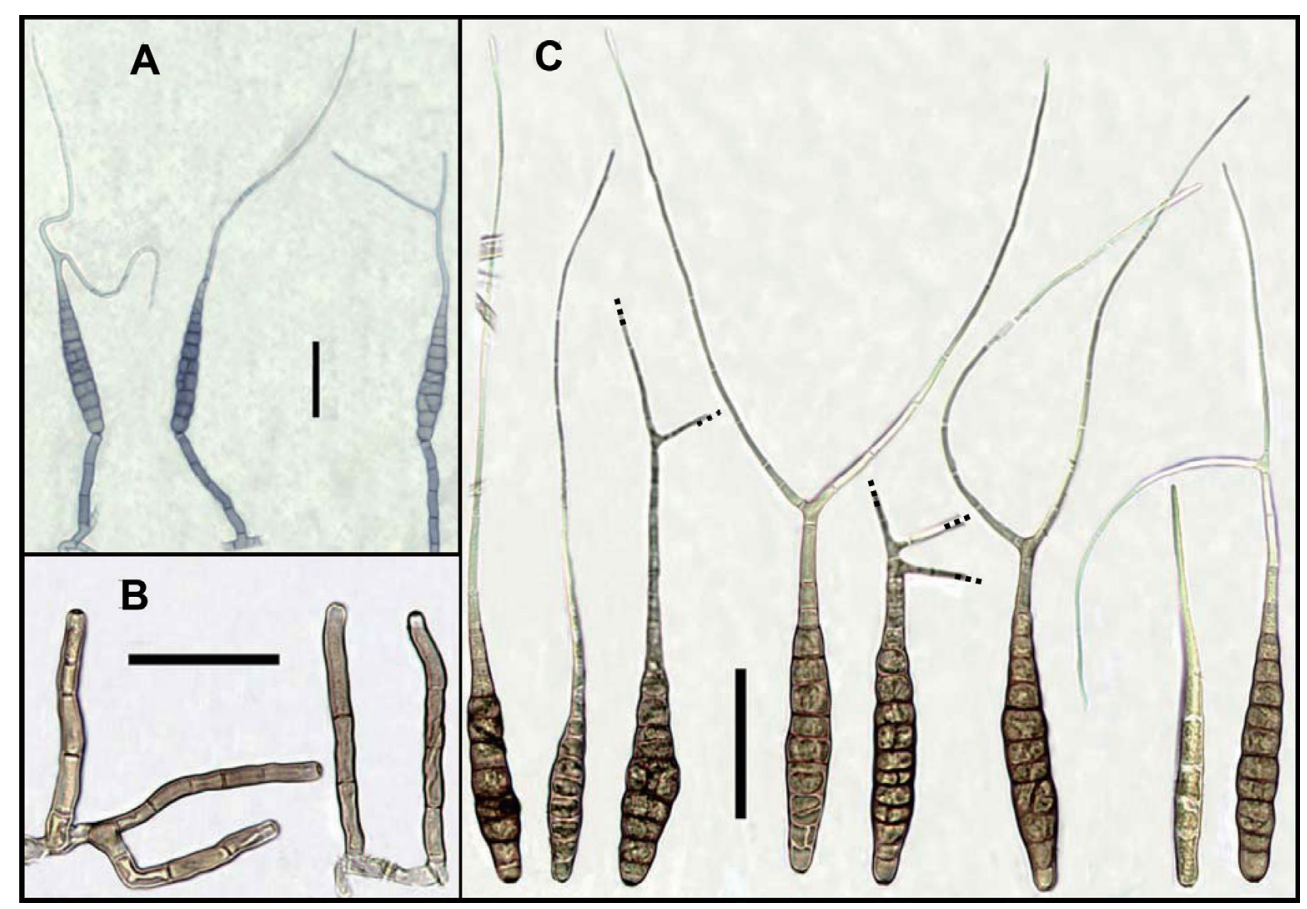

Fig. 2. Conidial morphology of the present isolate CNU 111459 from Cosmos bipinnatus. (A) sporulation patterns, (B) conidiophores, (C) conidia. Scale bars $=50 \mu \mathrm{m}$. 
selected conidia (50) from 7-day-old cultures were used to obtain conidial measurements and photograph was taken using an OLYMPUS BX50 light microscope (OLYMPUS, Tokyo, Japan) with an Artcam 300MI digital camera (ARTRAY, Tokyo, Japan).

The colonies on PDA were cotton or woolly, effuse, white or pale to dark grey; reverse yellow to orange, secreting a yellow pigment into the medium, $65-74 \mathrm{~mm}$ in diameter (Fig. 1B). Conidiophores on PCA were simple, erect or curved, septate (1-7), pale brown, smooth-walled, usually only one pigmented terminal conidiogenous site, measuring 25-120 $(-140) \times 4.5-8.5 \mu \mathrm{m}$ (Fig. 2A, 2B). Conidia were solitary, straight or slightly curved, mostly long obclavate, olivaceous brown to dark yellowish brown, smooth-walled, rostrate, tapering towards a long beak; conidium body $60-130(-140) \times 5-15(-22) \mu \mathrm{m}$, with $2-13$ (-16) transeverse septa, 1-4 longitudinal septa; beaks narrowly filamentous, filiform, sub hyaline to pale brown, often branched once, twice or more, 50-200 (-220) $\mu \mathrm{m}$ long, 2-3 $\mu \mathrm{m}$ wide, with $0-8$ septa (Fig. 2C).

The cultural, conidial and sporulation characteristics of the present Alternaria species was identical with the species described by Cho (2004) in Korea and $A$. cosmosa reported by $\mathrm{Wu}$ and $\mathrm{Li}$ in Taiwan (2005) even though there were some variation in the size of conidia (Table 1). Some morphological characteristics of Alternaria (e.g., color, size, shape of conidia, and sporulation patterns) that are used for identification sometimes overlap among species and vary depending on the cultural conditions (e.g., temperature and substrate) (Simmons and Roberts, 1993).

The first report of $A$. cosmosa was from cosmos seeds (Wu and Li, 2005). Richardson (1979) also reported another Alternaria species, A. zinniae, from cosmos seeds. But $A$. zinniae is easily differentiated from the present Alternaria species by producing unbranched beak. Morphologically, the present Alternaria species was distinct to $A$. carthami and similar with $A$. agripestis and
Table 1. Alternaria species used for phylogenetic analyses

\begin{tabular}{|c|c|c|c|}
\hline \multirow{2}{*}{ Species } & \multirow{2}{*}{ Isolate } & \multicolumn{2}{|c|}{ GenBank accession No. } \\
\hline & & Alt a 1 & gpd \\
\hline A. agripestis & CBS 577.94 & JQ646440 & JQ646356 \\
\hline A. carthami & CBS 635.80 & JQ646421 & JQ64633 \\
\hline A. cichorii & CBS 102.33 & JQ646424 & JQ646340 \\
\hline A. cirsinoxia & CBS 113261 & JQ646435 & JQ646351 \\
\hline A. cosmosa & CNU 3437 & KM655806 & KM655808 \\
\hline A. cosmosa & CNU 111459 & KM655807 & KM655809 \\
\hline A. cretica & CBS 109164 & JQ646426 & JQ646342 \\
\hline A. cyphomandrae & CBS 109155 & JQ646444 & JQ646360 \\
\hline A. danida & EGS 07-029 & JQ646420 & JQ646336 \\
\hline A. dichondrae & CBS 199.74 & JQ646441 & JQ646357 \\
\hline A. grandis & CBS 109158 & JQ646425 & JQ646341 \\
\hline A. hawaiiensis & CBS 630.93 & JQ646436 & JQ646352 \\
\hline A. linicola & CBS 103.46 & JQ646447 & JQ646363 \\
\hline A. multirostrata & CBS 712.68 & JQ646446 & JQ646362 \\
\hline A. passiflorae & CBS 113.38 & JQ646437 & JQ646353 \\
\hline A. porri & ATCC 58175 & AY563296 & AY278806 \\
\hline A. protenta & CBS 116696 & JQ646419 & JQ646335 \\
\hline A. scorzonerae & CBS 478.83 & JQ646418 & JQ646334 \\
\hline A. solani & EGS 44-098 & GQ180096 & GQ180080 \\
\hline A. solani-nigri & CBS 113403 & JQ646417 & JQ646333 \\
\hline A. subcylindrica & CBS 109161 & JQ646429 & JQ646345 \\
\hline A. tomatophila & EGS 42-156 & GQ180101 & GQ180085 \\
\hline
\end{tabular}

A. passiflorae (Table 2). However, A. agripestis differed from the present species by having ornamented conidial body, and $A$. passiflorae distinguished from the present species by having conidiophores with 4 to 5 conidiogenous loci (Simmons, 2007). Simmons (2007) mentioned that $A$. cosmosa and $A$. danida are morphologically similar species. However, conidial beak tip of $A$. danida is possible to convert into a secondary conidiophore yielding a chain of two conidia, the morphological phenomenon was not observed among the present $A$. cosmosa isolates, and not

Table 2. Morphological comparison among the present Alternaria species and the other related species

\begin{tabular}{|c|c|c|c|c|c|c|}
\hline \multirow{2}{*}{ Species } & \multicolumn{2}{|c|}{ Conidial body } & \multirow{2}{*}{ Beak $(\mu \mathrm{m})$} & \multirow{2}{*}{ Culture media } & \multirow{2}{*}{ Temperature } & \multirow{2}{*}{ References } \\
\hline & Size $(\mu \mathrm{m})$ & Transverse septa & & & & \\
\hline A. agripestis & $80-110 \times 11-18$ & $8-11$ & $190-260$ & PCA & $22^{\circ} \mathrm{C}$ & Simmons (2007) \\
\hline A. carthami & $75-90 \times 17-22$ & $8-10$ & $150-200$ & V8A & $22^{\circ} \mathrm{C}$ & Simmons (2007) \\
\hline A. danida & $95-120 \times 23-26$ & $6-11$ & $175-250(>250)$ & V8A & $22^{\circ} \mathrm{C}$ & Simmons (2007) \\
\hline CNU 111459 & $60-130(-141) \times 5-15(-22)$ & $2-13(-16)$ & $50-200(-220)$ & PCA & $22^{\circ} \mathrm{C}$ & In the study \\
\hline CNU 3437 & $50-100 \times 12-18$ & $6-13$ & $75-163$ & V8A & $20^{\circ} \mathrm{C}$ & Cho (2004) \\
\hline \multirow[t]{2}{*}{ A. cosmosa } & $50-110 \times 15-25$ & $7-12$ & $112.5-287.5$ & V8A & $25^{\circ} \mathrm{C}$ & Wu and $\mathrm{Li}$ (2005) \\
\hline & $50-100 \times 15-22.5$ & $6-11$ & $137.5-287.5$ & PCA & $25^{\circ} \mathrm{C}$ & Wu and $\mathrm{Li}(2005)$ \\
\hline A. passiflorae & $70-115 \times 16-22$ & $7-10(>10)$ & $100-220$ & V8A & $22^{\circ} \mathrm{C}$ & Simmons (2007) \\
\hline
\end{tabular}




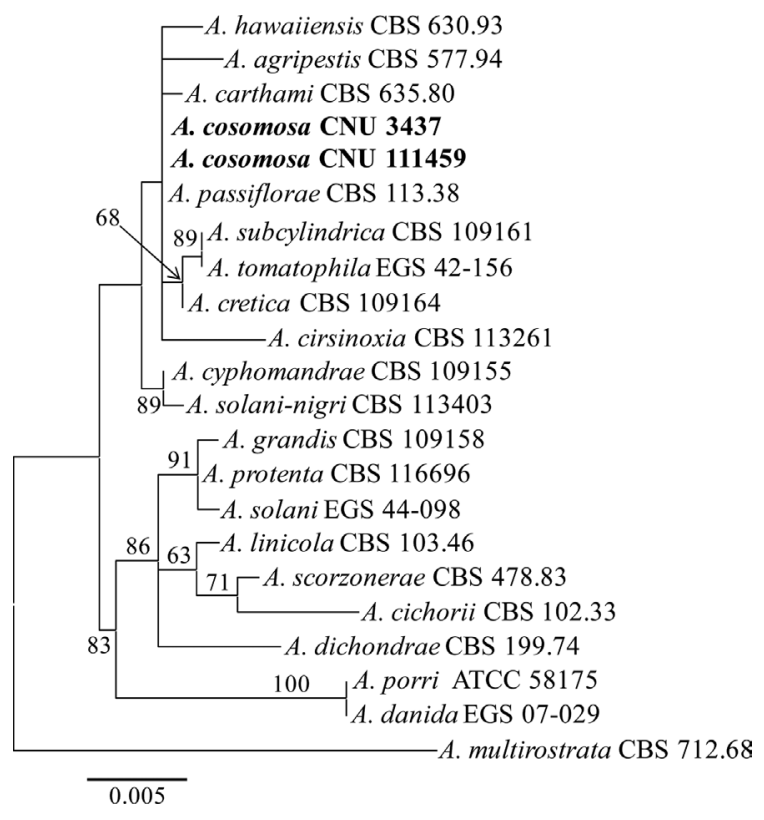

Fig. 3. Maximum likelihood tree of the present Alternaria species from Cosmos bipinnatus based on a combined dataset of Alt a1 and gpd gene sequences. Bootstrap values are shown above branches. The bar indicates nucleotide substitutions per site.

described by Wu and $\operatorname{Li}(2005)$.

Phylogenetic analysis. Genomic DNA of the present isolates (CNU 3437 and CNU 111459) was extracted using a modified protocol of Park et al. (2005). Fragments containing Alternaria allergen a 1 (Alt a1) gene and glyceraldehyde-3-phosphate dehydrogenase $(g p d)$ gene were amplified with primers Alt-a1-for \& Alt-a1-rev (Hong et al., 2005), and gpd 1 \& gpd2 (Berbee et al., 1999), respectively. PCR products were purified using a Wizard PCR prep kit (Promega, Madison, WI, USA) and sequenced using commercial sequencing service provider (Macrogen, Seoul, Korea). Resultant sequences were deposited in GenBank (Table 1), from which the molecular sequences for the other species were retrieved and included in the study. A pair-wise alignment of all sequences was completed using the ClustalX program (Thompson et al., 1997). Phylogenetic relationships of a combined dataset were estimated by a maximum likelihood analysis using the RAxML program (Stamatakis et al., 2008).

PCR amplification resulted DNA fragments in size of 452 and $564 \mathrm{bp}$ from Alt a1 and gpd genes, respectively. The present Alternaria isolates CNU 111459, CNU 3437 and $A$. passiflorae could not be differentiated by the sequences of these two genes (Fig. 3). Phylogenetic analysis of the dataset revealed that the present Alternaria species was closely related to $A$. carthami and $A$. agripestis, genetically distant to $A$. danida which was accordance to the results of Lawrence et al. (2013).

Although the present Alternaria species could be delimited from A. passiflorae based on the phylogenetic analysis in the study, the morphology of conidial body and condiophore was an obvious delimitation characteristic. Hence, the present Alternaria isolates CNU 111459 and CNU 3437 were all confirmed as Alternaria cosmosa Y.L. $\mathrm{Li} \&$ W.S. Wu (2005). This is the first report of Alternaria blight caused by A. cosmosa on Cosmos bipinnatus in Korea.

Pathogenicity. The pathogenicity of the species was conducted by spraying conidial suspension $\left(1 \times 10^{5}\right.$ conidia/ $\mathrm{ml})$ to the detached surface-sterilized leaves. Necrotic spots were induced after two to three days and enlarged the size with age (data not shown). Conidia of $A$. cosmosa were re-isolated from the diseased leaves. A. cosmosa were pathogenic to Cosmos bipinnatus and C. sulphureus (yellow cosmos), which is readily infected the lower leaves of yellow cosmos and caused brownish spots (Wu et al., 2007). During our observation in Korea, the causal disease was also commonly found on the lower leaves of $C$. bipinnatus.

\section{Acknowledgment}

This study was supported by a grant from the National Institute of Biological Resources (NIBR), funded by the Ministry of Environment (MOE) of the Republic of Korea.

\section{References}

Berbee, M. L., Pirseyedi, M. and Hubbard, S. 1999. Cochliobolus phylogenetics and the origin of known, highly virulent pathogens, inferred from ITS and glyceraldehyde-3-phosphate dehydrogenase gene sequences. Mycologia 91:964-977.

Cho, H. S. 2004. Taxonomic studies on Korean species of $\mathrm{Al}$ ternaria based on morphology and molecular phylogenetic analysis. Ph.D. Thesis. Chungnam National University, Deajeon, Korea.

Dubey, S. and Singh, V. K. 2011. Population dynamics of Aphis spiraecola Patch (Homoptera: Aphididae) on medicinal plant Cosmos bipinnatus in eastern Uttar Pradesh, India. Adv. Life Sci. 1:54-58.

Hong, S. G., Cramer, R. A., Lawrence, C. B. and Pryor, B. M. 2005. Alt a 1 allergen homologs from Alternaria and related taxa: analysis of phylogenetic content and secondary structure. Fungal Genet Biol. 42:119-129.

Jang, I. C., Park, J. H., Park, E., Park, H. R. and Lee, S. C. 2008. 
Antioxidative and antigenotoxic activity of extracts from cosmos (Cosmos bipinnatus) flowers. Plant Foods Hum Nutr. 63:205-210.

Lawrence, D. P., Gannibal, P. B., Peever, T. L. and Pryor, B. M. 2013. The sections of Alternaria: formalizing species-groups concepts. Mycologia 105:530-546.

Park, S. H. 2009. New Illustrations and Photographs of Naturalized Plants of Korea. Ilchokok Publisher, Seoul. Korea. 575 pp. (in Korean)

Park, J. H., Cho, S. E., Park, M. J., Hong, S. H. and Shin, H. D. 2012. Occurrence of white smut caused by Entyloma cosmi on Cosmos bipinnatus in Korea. Res. Plant Dis. 18:250-254 (in Korean).

Park, M. S., Seo, G. S., Bae, K. S. and Yu, S. H. 2005. Characterization of Trichoderma spp. associated with green mold of Oyster mushroom by PCR-RFLP and sequence analysis of ITS regions of rDNA. Kor. J. Plant Pathol. 21:229-236.

Richardson, M. J. 1979. An annotated list of seed-borne disease.
CAB International, Surrey, UK. 320 pp.

Simmons, E. G. 2007. Alternaria: An Identification Manual. CBS Fungal Biodiversity Centre, Utrecht, Netherlands.

Simmons, E. G. and Roberts, R. G. 1993. Alternaria themes and variations (73). CBS Fungal Mycotaxon 48:109-140.

Stamatakis, A., Hoover, P. and Rougemont, J. 2008. A rapid bootstrap algorithm for the RAxML Web servers. Syst. Biol. 57:758-771.

Thompson, J. D., Gibson, T. J., Plewniak, F., Jeanmougin, F. and Higgins, D. G. 1997. The CLUSTAL_X windows interface: flexible strategies for multiple sequence alignment aided by quality analysis tools. Nucleic Acids Res. 25:4876-4882.

Wu, W. S. and Li, Y. L. 2005. A new species of Alternaria on cosmos seeds. Mycotaxon 91:15-20.

Wu, W. S., Li, Y. L. and Wu, H. C. 2007. Potential of Bacillus amyloliquefaciens for control of Alternaria cosmosa and $A$. patula of Cosmos sulfurous (Yellow Cosmos) and Tagetes patula (French Marigold). Phytopathology 155:670-675. 